\title{
Efectos de un programa de intervención sobre el desarrollo del pensamiento crítico en universitarios chilenos
}

\author{
Effects of an intervention program on the development of critical thinking in chilean university students
}

DOI: https://doi.org/10.21803/penamer.14.27.375

Sonia Betancourth Zambrano orcid.org/0000-0003-1651-085X

Viviana Martínez Daza orcid.org/0000-0001-5951-5250

Yuranny Tabares Díaz https://orcid.org/0000-0002-1717-6710

Ana Castillo Leyton https://orcid.org/0000-0003-0231-7135

¿Cómo citar este artículo?

Betancourth, S., Martínez, V., Tabares, Y. \& Castillo, A. (2021). Efectos de un programa de intervención sobre el desarrollo del pensamiento crítico en universitarios chilenos. Pensamiento Americano, 14(27), 125-136

DOI: https://doi.org/10.21803/penamer.14.27.375

\section{Resumen}

Introducción: el pensamiento crítico es un proceso cognitivo orientado a la interpretación, inferencia, evaluación, análisis y reflexión de las situaciones del contexto, a través del conocimiento y la inteligencia, para llegar de forma efectiva a la posición más razonable y justificada sobre un tema. Objetivo: analizar los efectos de un programa de intervención basado en meeting en cadena, triangulación transformadora y controversia sobre el pensamiento crítico en estudiantes de la carrera de trabajo social de una universidad chilena. Materiales y Métodos: el estudio se enmarca en el paradigma cuantitativo, de diseño cuasiexperimental con preprueba y posprueba con un solo grupo. La muestra se conformó por 33 estudiantes universitarios. El programa de intervención se constituyó de un total de seis sesiones, dentro de las cuales se implementaron tres estrategias de desarrollo de pensamiento crítico correspondientes a meeting en cadena, triangulación transformadora y controversia; así también, se incluyó asesorías presenciales y virtuales. Resultados: se encontró que existen efectos positivos en el pensamiento crítico de los estudiantes participantes, posterior al desarrollo del programa de intervención. Conclusiones: las estrategias de desarrollo de pensamiento crítico promueven la construcción del conocimiento, la capacidad de adopción de una postura, la autoevaluación del propio pensamiento y el reconocimiento de puntos de vista divergentes.

Palabras clave: Pensamiento crítico; Estrategias de desarrollo; Estudiantes universitarios.

\begin{abstract}
Introduction: critical thinking is a cognitive process oriented to the interpretation, inference, evaluation, analysis and reflection of context situations, through knowledge and intelligence, to arrive effectively at the most reasonable and justified position on an issue. Objective: to analyze the effects of an intervention program based on chain meeting, transformative triangulation and controversy about critical thinking in students of the social work career of a Chilean university. Materials and Methods: the study is framed in the quantitative paradigm, of quasiexperimental design with preprogramming and posttesting with a single group. The sample consisted of 33 university students. The intervention program consisted of a total of six sessions, in which three critical thinking development strategies corresponding to chain meeting, transformative triangulation and controversy were implemented; It also included faceto-face and virtual counseling. Results: it was found that there are positive effects on the critical thinking of the participating students, after the
\end{abstract}


development of the intervention program. Conclusions: Critical thinking development strategies promote the construction of knowledge, the ability to adopt a posture, the self-assessment of one's own thinking and the recognition of divergent points of view.

Keywords: Critical thinking; Strategies for development; University students.

\section{Resumo}

a

Keywords: a

\section{Perfiles}

Doctora en Psicología Escolary Desarrollo, Departamento de Psicología, Universidad de Nariño, Pasto-Colombia. Correo: sbetan@gmail.com

Estudiante de Especialización en gerencia de la seguridad y salud en el trabajo, Universidad de Nariño, Pasto-Colombia. Correo: vivianaamd@gmail.com

Estudiante de Maestría en Promoción y Prevención en Salud Mental, Departamento de Psicología, Universidad de Nariño. Correo: yuranny.tabares06@gmail.com

Magister en Educación, Departamento de Trabajo Social, Universidad de Atacama, Atacama-Chile. Correo: ana.castillo@uda.cl
Sonia Betancourth Zambrano

Viviana Martínez Daza

Yuranny Tabares Díaz

Ana Castillo Leyton 


\section{Introducción}

El pensamiento crítico es definido por Paul y Elder (2003) como un proceso que permite al individuo apoderarse de las estructuras propias del acto de pensar, sometiéndolas a estándares intelectuales, que demandan la formulación de preguntas con precisión y claridad, la evaluación de la información obtenida y el establecimiento de soluciones y conclusiones con base en un juicio autodirigido, auto disciplinado, autorregulado y autocorregido, elementos que en su conjunto mejoran la calidad del pensamiento.

En este marco emerge la relevancia del pensamiento crítico en la educación superior, dado que constituye un aspecto clave para la consecución de una sociedad sostenible, cuyo eje central sea la educación para la vida y el ejercicio de una acción transformadora que impacte a corto y largo plazo las esferas educativa, profesional, personal y social del sujeto (Bezanilla et al., 2018; Henríquez et al., 2019).

En el contexto actual se han realizado diferentes investigaciones tendientes a evaluar el pensamiento crítico en población estudiantil, tal es el caso del estudio de Robles et al. (2016) en universitarios de pregrado y posgrado de México, el cual reporta niveles bajos de este atributo en las dimensiones de razonamiento, toma de decisiones y solución de problemas. De igual manera, se encuentra un ejercicio investigativo desarrollado en la Costa Caribe Colombiana, cuyos resultados dan cuenta de la existencia de falencias en los niveles de pensamiento crítico en estudiantes de cuarto a sexto semestre de Licenciaturas en Educación (Steffens et al., 2018).

La investigación de Macedo (2018) en estudiantes peruanos de la Facultad de Ingeniería Económica, Estadística y Ciencias Sociales, evidenció la prevalencia de niveles medios y bajos de pensamiento crítico, particularmente en la habilidad de inferencia. De modo semejante, los datos de un estudio en universitarios de Perú de la Facultad de Educación, indicó que más de la mitad de la muestra evaluada se ubicó en un nivel medio de pensamiento crítico (Pineda y Cerrón, 2015).

Específicamente en Chile, Betancourth, et al. (2017) muestran los resultados de una investigación llevada a cabo con estudiantes de Derecho, arrojando como principales hallazgos la presencia de niveles bajos de desarrollo de habilidades de pensamiento crítico. Paralelamente, se halla un estudio sobre evaluación del pensamiento crítico y científico en estudiantes de Pedagogía de una universidad chilena, el cual concluyó que el nivel de desempeño global de dicho atributo, presentó una media relativamente baja en contraste con el puntaje máximo de la prueba aplicada (Ossa et al., 2018).

Lo expuesto anteriormente, refleja que los niveles de pensamiento crítico en los universitarios se ubican en rangos medios y bajos, visibilizando con ello la existencia de limitaciones e insuficiencias en la educación superior frente al desarrollo de las habilidades que comprende y, por ende, la necesidad de realizar cambios en los sistemas educativos en aras de revertir dicha problemática (Palacios et al., 2017).

Resulta relevante reconocer que en la actualidad pensar críticamente se torna esencial, puesto que permanentemente la sociedad enfrenta crisis en sus dimensiones social, política, económica, cultural, entre otras, demandando por parte de los sujetos el desarrollo de habilidades cognitivas para la consecución de la autonomía de su pensamiento (Jaimes, 2016). Es así como Tünnermann (2003) sostiene que: "Las tendencias innovadoras que hoy día se observan en la educación superior no pueden sustraerse de la influencia de los dos fenómenos que más inciden en su desempeño: la globalización y la emergencia de las sociedades del conocimiento" (p.108).

Es de señalar que se ha impulsado desde distintos ámbitos un proceso de discusión en torno a la calidad de la educación impartida en las aulas universitarias, desencadenando con ello la renovación de las metodologías educativas, pasando a centrarse en la adopción de un enfoque basado en competencias,

Pensamiento Americano Vol. 14 - No. 27 - p.p. 125-136 • 2021 • Enero-Junio Corporación Universitaria Americana • Barranquilla, Colombia • ISSN-e: 2745-1402 
tendiente a articular los procesos formativos con las necesidades del entorno local, nacional e internacional, a fin de impactar en el rol ejercido por los docentes y estudiantes (Bezanilla et al., 2018).

No obstante, en Chile una de las mayores dificultades reportadas en la implementación de este tipo de modelos educativos alude a la heterogeneidad de competencias presentes en los estudiantes que ingresan a la universidad, razón por la cual uno de los retos ineludibles consiste en acortar dichas brechas, mediante el diseño e implementación de un sistema curricular que brinde respuesta a los lineamientos de la normativa legal vigente. Lo planteado, con el objetivo de propender por una formación integral que faculte a los universitarios en la adquisición de conocimientos y fortalecimiento de competencias genéricas y específicas, exigibles para su posterior inserción en el mundo laboral (Villarroel y Bruna, 2014).

Desde esta perspectiva, la carrera de trabajo social de una universidad chilena ha emprendido como iniciativa la implementación de una nueva malla curricular basada en competencias y circunscrita bajo un sello de pensamiento crítico, como elemento transversal en la formación de sus estudiantes. Si bien esto constituye un avance importante en la formación universitaria chilena, aún se vislumbra la necesidad de realizar innovaciones a nivel metodológico y curricular, que trasciendan las aulas y generen aprendizajes desde la interacción en espacios sociales dinámicos que conduzcan al autoconocimiento, autorreconocimiento de estilos de aprendizaje y establecimiento de metas educativas tangibles (Espinosa y Estévez, 2013; Palacios et al., 2017).

Para lograr el desarrollo de estas habilidades y disposiciones propias del pensamiento crítico, existen técnicas y estrategias de enseñanza, tales como, la discusión socrática (Betancourth et al., 2012), el debate crítico (Fuentes, 2011), el aprendizaje basado en problemas (Guevara, 2010), el análisis de textos, noticias y medios de comunicación (Montoya y Monsalve, 2008), así como las dispuestas para este estudio dentro de las cuales se incluyen meeting en cadena, triangulación transformadora y controversia.
Bajo esta línea, se han llevado a cabo programas de desarrollo del pensamiento crítico en países como España (Saiz y Rivas, 2011), Colombia (Betancourth et al., 2013, 2020) y Chile (Lazo y Herrera, 2011; Núñez et al., 2017), orientados a analizar los efectos de propuestas de intervención enmarcadas en estrategias de argumentación, decisión, resolución de problemas en situaciones diarias, controversia-socrática, debate crítico, pedagogía y aprendizaje basado en problemas, respectivamente, los cuales evidencian de manera común resultados positivos frente al incremento del nivel de pensamiento crítico en los estudiantes evaluados. No obstante, aún prevalecen vacíos de conocimiento en este campo de estudio, que permitan dar cuenta de propuestas para el fortalecimiento de este proceso cognitivo y a la par evalúen su efecto en el pensamiento crítico en población estudiantil universitaria.

\section{OBJETIVO}

Analizar los efectos de un programa de intervención basado en meeting en cadena, triangulación transformadora y controversia sobre el pensamiento crítico en estudiantes de la carrera de trabajo social de una universidad chilena.

\section{METODOLOGÍA}

\section{Tipo de estudio y diseño}

El estudio fue de tipo cuantitativo, empleando un diseño cuasi experimental con preprueba y posprueba con un solo grupo, dado que consideró un grupo de participantes predeterminado, en este caso, estudiantes matriculados a segundo año de la carrera de trabajo social de una universidad chilena (Hernández et al., 2014).

\section{Variables de estudio}

Variable dependiente: El pensamiento crítico, constructo que se define como un proceso cognitivo orientado a la interpretación, inferencia, evaluación, análisis y reflexión de las situaciones del con-

Pensamiento Americano Vol. 14 - No. 27 - p.p. 125-136 • 2021 • Enero-Junio Corporación Universitaria Americana • Barranquilla, Colombia • ISSN-e: 2745-1402 http://publicaciones.americana.edu.co/index.php/pensamientoamericano/index 
texto, a través del conocimiento y la inteligencia, para llegar de forma efectiva a la posición más razonable y justificada sobre un tema (Bernal et al., 2019; Facione, 2007). Se evaluó a través de la Prueba de Pensamiento Crítico antes y después del programa de intervención.

Variable independiente: El programa de intervención, integrado por tres estrategias de desarrollo de pensamiento crítico correspondientes a meeting en cadena, triangulación transformadora y controversia, las cuales se implementaron en el marco de la asignatura: Habilidades y destrezas para el trabajo social.

\section{Participantes}

El proceso investigativo se desarrolló con un total de 33 estudiantes de segundo año de la carrera de trabajo social de una universidad chilena. Se realizó un muestreo no probabilístico por conveniencia, acorde a la intención declarada de los estudiantes de participar en el estudio.

\section{Instrumento de recolección de información}

Se empleó como instrumento de medición la versión adaptada y validada para el contexto chileno de la Prueba de Pensamiento Crítico (Betancourth et al., 2018).

El análisis factorial de la prueba arrojó un único factor, denominado pensamiento crítico. En cuanto a las propiedades psicométricas, la confiabilidad del instrumento evaluada a través de alfa de Cronbach fue de 0.90, indicativa de un elevado nivel de consistencia interna. Conjuntamente, se realizó validez de contenido a través del juicio de expertos y validez de constructo mediante la aplicación de la Escala de Autoestima de Rosenberg (Betancourth et al., 2018).

El instrumento contiene 27 afirmaciones con respuesta cerrada, cuyos valores categoriales se hallan en una escala de frecuencia de 1 a 5 , en la cual 1 corresponde a nunca, 2 a rara vez, 3 a algunas veces, 4 a casi siempre y 5 a siempre. La identificación del nivel de pensamiento crítico de los estudiantes evaluados, se realizó a partir de la distribución de los puntajes en 5 categorías, estas son: muy bajo (27 a 88 puntos), bajo (89 a 96 puntos), medio (97 a 106 puntos), alto (107 a 113 puntos) y muy alto (114 a 135 puntos) (Betancourth et al., 2018). Así también, se aplicó una ficha de caracterización de datos sociodemográficos de los participantes.

\section{Procedimiento}

Se solicitó en primera instancia una autorización por escrito a los estudiantes para participar del estudio, a través de un formato de consentimiento informado. Seguidamente, se llevó a cabo la aplicación del pre-test de la Prueba de Pensamiento Crítico. Posteriormente, se desarrolló el programa de intervención, con una extensión total de seis sesiones; finalmente, una semana después del programa, se aplicó el post-test. La prueba se administró en formato papel y lápiz.

El programa de intervención integró la implementación de tres estrategias de desarrollo de pensamiento crítico correspondientes a: meeting en cadena, consiste en la construcción de argumentos compartidos sobre un tema, reconociendo y retomando el relato de otra persona para anclarlo a los argumentos propios (Vilà et al., 2005); triangulación transformadora, retoma el ensayo como medio para la evaluación y construcción de argumentos, incorporando la postura personal, base teórica y elementos éticos/ políticos/legales (Álvarez, 2013); controversia, hace referencia al proceso de adoptar una posición y discutirla, para posteriormente cambiarla, establecer acuerdos y sintetizar los argumentos expuestos (Betancourth et al., 2019).

El programa incluyó la realización de asesorías presenciales y virtuales a través del uso de TICs (correo, redes sociales y curso virtual en Moodle), organizadas en horarios acordes a la disponibilidad de los participantes.

Meeting en cadena: Se llevó a cabo una sesión para la aplicación de la estrategia de pensamiento

Pensamiento Americano Vol. 14 - No. 27 - p.p. 125-136 • 2021 • Enero-Junio Corporación Universitaria Americana • Barranquilla, Colombia • ISSN-e: 2745-1402 
crítico de meeting en cadena, en torno a la temática ontología del lenguaje. Para ello, se desarrollaron las siguientes etapas: a) socialización de la metodología de la estrategia; b) asignación al azar de preguntas orientadoras; c) construcción de argumentos; d) plenaria de argumentos; y e) retroalimentación. La evaluación de la estrategia se efectuó por medio de una pauta de cotejo diseñada previamente por el equipo investigador y el feedback brindado a cada equipo de trabajo.

Triangulación transformadora: Se desarrolló una sesión para la aplicación de la estrategia de pensamiento crítico de triangulación transformadora, enmarcada en la temática lenguaje y acción, siguiendo las etapas descritas a continuación: a) socialización de la metodología de la estrategia; b) elaboración del diagrama de triangulación (postura personal, base teórica y elementos éticos, políticos y legales); c) plenaria; y d) retroalimentación. La evaluación de la estrategia se realizó mediante una rúbrica construida por los investigadores y el feedback brindado a los equipos de trabajo.

Controversia: Se emplearon dos sesiones para la aplicación de la estrategia de controversia enfocada en la temática, teorías de desarrollo del lenguaje. De este modo, se realizaron las siguientes etapas en la sesión 1: a) socialización de la metodología de la estrategia; b) asignación de posturas; y c) entrega de material de lectura y elaboración de argumentos. Por su parte, en la sesión 2 se llevaron a cabo estas etapas: a) debate inicial; b) intercambio de posiciones; y c) retroalimentación. El proceso de evaluación de la estrategia se efectuó mediante rúbricas y pautas de cotejo estructuradas por los investigadores y el feedback proporcionado a los equipos de trabajo.

\section{Análisis de datos}

Se llevó a cabo un análisis descriptivo de las variables sociodemográficas correspondientes a sexo, edad, pertenencia a pueblos originarios o indígenas, estrato socioeconómico, procedencia de institución educacional y puntaje de la Prueba de Selección Uni- versitaria (PSU). Posteriormente, se realizó un análisis descriptivo de los puntajes obtenidos en la aplicación de la Prueba de Pensamiento Crítico, a fin de identificar el nivel de pensamiento crítico de los participantes en el pre-test y post-test y determinar la existencia de diferencias estadísticamente significativas entre dichas mediciones. Por último, se efectuó un contraste entre los resultados de la prueba y las variables sociodemográficas de los participantes, para evidenciar la presencia de relaciones entre las mismas.

\section{Resultados}

La investigación tuvo como propósito determinar la efectividad de un programa de intervención basado en estrategias tales como: meeting en cadena, triangulación transformadora y controversia sobre el desarrollo del pensamiento crítico en estudiantes de la carrera de trabajo social de una universidad chilena. En este orden de ideas, se presentan a continuación los resultados de la caracterización de los participantes, así como los hallazgos derivados del programa implementado.

\section{Caracterización de los participantes}

La muestra estuvo conformada por 33 estudiantes pertenecientes a segundo año de la carrera de trabajo social de una universidad chilena, de los cuales 24 eran mujeres $(72.7 \%)$ y 9 hombres (27.3 $\%$ ), cuyas edades fluctuaban entre los 19 a 30 años (media= 21.03 años). Asimismo, el 39.4\% de los participantes señaló pertenecer a pueblos originarios o indígenas y el $60.6 \%$ la no pertenencia a los mismos.

Por otro lado, el $54.5 \%$ de los estudiantes indicó que proviene de una institución de educación municipal y un $45.5 \%$ de una institución particular subvencionada. En relación con los resultados obtenidos por los estudiantes en la Prueba de Selección Universitaria (PSU), la cual constituye un requisito de ingreso a las Instituciones de Educación Superior del país, los puntajes para la carrera de Trabajo Social se ubicaron en un $24 \%$ en un rango de 451 a 550 puntos, seguidos de un $58 \%$ con puntuaciones

Pensamiento Americano Vol. 14 - No. 27 - p.p. 125-136 • 2021 • Enero-Junio Corporación Universitaria Americana • Barranquilla, Colombia • ISSN-e: 2745-1402 http://publicaciones.americana.edu.co/index.php/pensamientoamericano/index 
superiores a 551, en una escala estándar de 150 a 850 puntos, tal como se expone en la figura 1.

\section{Figura 1.}

Puntajes PSU.

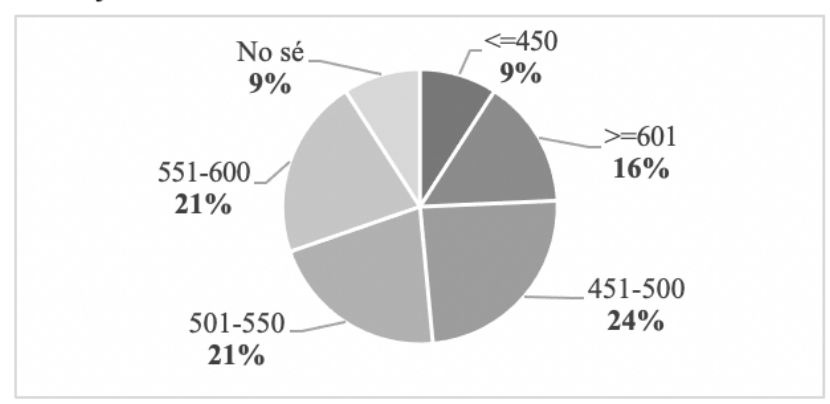

Efectos del programa de intervención sobre el pensamiento crítico

En primer lugar, se realizó un análisis descriptivo de los resultados obtenidos en la pre y posprueba a fin de reconocer el nivel de pensamiento crítico de los estudiantes en dichas mediciones. Posteriormente, se aplicó la prueba de contraste de normalidad de Shapiro-Wilk para muestras inferiores a 50 casos, la cual arrojó un p-valor de 0.03 indicando que el conjunto de datos no presenta una distribución normal; a partir de ello, se utilizó la prueba no paramétrica de Wilcoxon para identificar la existencia de diferencias significativas entre la pre y posprueba, empleando paralelamente los rangos que arroja la misma para determinar si son positivos, negativos o neutros.

Por último, se hizo uso de la prueba de correlación Rho de Spearman para evidenciar la existencia de relaciones entre los puntajes totales obtenidos en la Prueba de Pensamiento Crítico y las variables sociodemográficas. Los resultados fueron los siguientes:

La figura 2 muestra que 7 estudiantes obtuvieron un nivel de pensamiento crítico muy alto en la preprueba, 9 estudiantes nivel alto, 7 estudiantes nivel medio, 7 estudiantes nivel bajo y 3 estudiantes nivel muy bajo.

Por su parte, la figura 3 ilustra que 11 estudiantes presentaron un nivel de pensamiento crítico muy alto en la posprueba, 9 estudiantes nivel alto, 7 es-
Figura 2.

Resultados preprueba pensamiento crítico

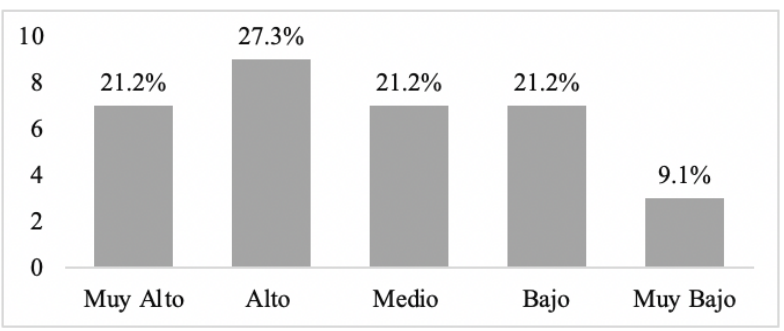

Figura 3.

Resultados posprueba pensamiento crítico.

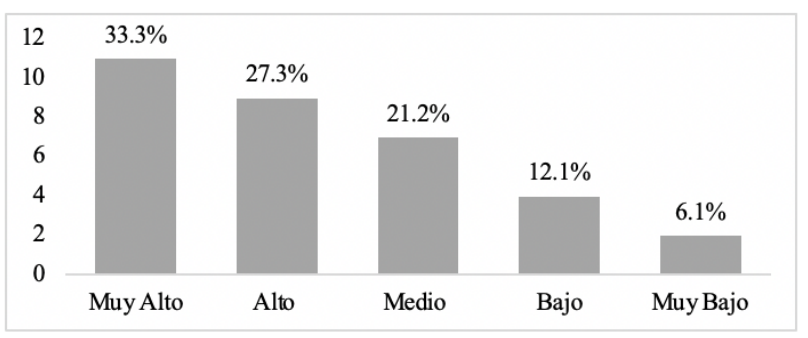

tudiantes nivel medio, 4 estudiantes nivel bajo y 2 estudiantes nivel muy bajo.

La figura 4 presenta el contraste de los resultados obtenidos en la medición pre y post de la prueba en cuanto a los niveles de desarrollo de pensamiento crítico arrojados por la misma. Respecto al nivel muy alto hay un incremento en el número de estudiantes que obtuvieron dicho nivel en la posprueba frente a la preprueba. En el caso de los niveles altos y medio, se evidencia una regularidad en el número de personas que obtuvieron estos niveles en las mediciones realizadas. Finalmente, frente a los niveles bajo y muy bajo existe un descenso en el número de personas que presentaron estos niveles en la post prueba en comparación a la preprueba.

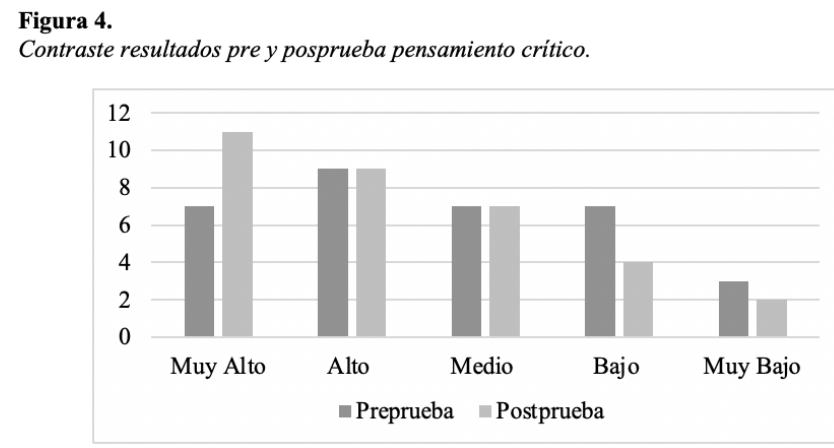


A partir los resultados obtenidos en la prueba de Wilcoxon, cuyo p-valor fue inferior a 0.05 , se evidencia que existen diferencias significativas en el pensamiento crítico entre la preprueba y la posprueba de los estudiantes, tal como se ilustra en la tabla 1.

Tabla 1.

Resultado de la prueba de Wilcoxon para el pensamiento crítico Post-prueba - Preprueba

Z $-2,273^{\mathrm{b}}$

Sig. asintótica $\quad, 023$

(bilateral)

En correspondencia a la tabla 2, se presentaron 22 de 33 rangos positivos, es decir, 22 estudiantes de 33 en total incrementaron su pensamiento crítico después del programa de intervención. Los datos que se encuentran dentro de la casilla de empates demuestran que un estudiante mantuvo el mismo puntaje. De la misma manera, se presentaron rangos negativos en los resultados, indicando que 10 estudiantes descendieron los puntajes obtenidos en la variable de estudio.

Tabla 2.

Rango de la prueba de Wilcoxon para el pensamiento crítico

\begin{tabular}{llccc}
\hline & $\mathrm{N}$ & $\begin{array}{c}\text { Rango } \\
\text { promedio }\end{array}$ & $\begin{array}{c}\text { Suma } \\
\text { de } \\
\text { rangos }\end{array}$ \\
\hline Post-prueba - Rangos negativos & $10^{\mathrm{a}}$ & 14,25 & 142,50 \\
Pre-prueba & Rangos positivos & $22^{\mathrm{b}}$ & 17,52 & 385,50 \\
& $\begin{array}{l}\text { Empates } \\
\text { Total }\end{array}$ & $1^{\mathrm{c}}$ & & \\
\hline
\end{tabular}

Por otro lado, al realizar la comparación de los resultados de la prueba con las variables sociodemográficas, se identifica que no hay correlaciones entre estos, dado que los p-valor arrojados por la prueba de correlación Rho de Spearman fueron mayores a 0.05 en todos los casos.

Finalmente, se puede concluir que existen efectos de la variable independiente sobre la variable dependiente, es decir, el pensamiento crítico de los estudiantes de segundo año de la Carrera de Trabajo Social aumentó con el programa de intervención desarrollado.

\section{CONCLUSIONES Y DISCUSIÓN}

La investigación tuvo por objetivo analizar los efectos de un programa de intervención basado las estrategias de meeting en cadena, triangulación transformadora y controversia sobre el pensamiento crítico en estudiantes de la carrera de trabajo social de una universidad chilena. Respecto a los resultados, se observan diferencias estadísticamente significativas entre las mediciones preprueba y posprueba, denotando la efectividad del programa en el desarrollo de destrezas intelectuales.

Lo anterior, se contrasta con estudios que de forma semejante implementaron estrategias de desarrollo de pensamiento crítico en población universitaria, como los llevados a cabo por Acosta (2002) y Betancourth et al. (2012; 2013), los cuales se orientaron a determinar la efectividad de la controversia-socrática en la adquisición y fortalecimiento de habilidades de este tipo. Dichas investigaciones concluyen que la estrategia es eficaz y promueve la construcción del conocimiento, la capacidad de adopción de una postura, la autoevaluación del propio pensamiento y el reconocimiento de puntos de vista divergentes frente a un tópico.

De la misma forma, se destacan estudios sobre programas de entrenamiento en pensamiento crítico que tuvieron como foco el uso de otras estrategias, como la discusión socrática (Betancourt, 2010), el aprendizaje basado en problemas (Hincapie et al., 2017; Núñez et al., 2017; Sánchez y Rivas, 2012; Sastoque et al., 2016) y el debate crítico (Betancourth et al., 2020; Leitão et al., 2016). Estos ejercicios investigativos señalan como principales hallazgos la presencia de mejoras significativas en los niveles de pensamiento crítico de las muestras participantes, posterior a los programas implementados y, por ende, la eficacia de la labor educativa frente a la ejecución de estas iniciativas.

Se puede considerar que las estrategias de pensamiento crítico realizadas en el presente estudio, las descritas en otros antecedentes y todas aquellas

Pensamiento Americano Vol. 14 - No. 27 - p.p. 125-136 • 2021 • Enero-Junio Corporación Universitaria Americana • Barranquilla, Colombia • ISSN-e: 2745-1402 http://publicaciones.americana.edu.co/index.php/pensamientoamericano/index 
que tengan como base fundamental el desarrollo del pensamiento crítico, suscitan la construcción de ambientes educativos en los cuales el estudiante es el agente activo de su proceso de aprendizaje, dinamizando a su vez las condiciones necesarias para que cada persona de forma particular, pero con base en elementos comunes para todos, alcance el desarrollo óptimo de habilidades que les permitan plantear juicios confiables y generar soluciones eficaces a los problemas del entorno en el que estén inmersas (González, 2006; Sastoque et al., 2016).

$\mathrm{Al}$ analizar los efectos del programa de intervención a partir del número de estudiantes que incrementaron, disminuyeron o mantuvieron sus puntajes en pensamiento crítico, se encontró que 22 estudiantes aumentaron, 10 estudiantes disminuyeron y l estudiante obtuvo la misma puntuación en la variable de estudio. Lo expuesto, podría deberse por un lado a que los estudiantes que disminuyeron su puntaje en la prueba de pensamiento crítico no asistieron a la totalidad de sesiones de intervención y, por otra parte, a que la baja motivación hacia la realización de las actividades propuestas en las estrategias del programa influyó en el no incremento del nivel de desarrollo del atributo.

Diferentes referentes teóricos (Ennis, 1996; Facione, 2007; Halpern, 1998; Paul y Elder, 2002) aluden que el desarrollo del pensamiento crítico depende de dos componentes, estos son: habilidades y disposiciones, los cuales son imprescindibles en el campo de estudio, puesto que si un individuo conoce las habilidades que debe poner en marcha en una determinada situación, pero no está motivado a hacerlo o su fin no es ético, no será un buen pensador crítico.

El abordaje de las disposiciones del pensamiento crítico se ha llevado a cabo desde enfoques distintos pero complementarios entre sí, integrando el análisis de las disposiciones como una motivación general hacia el pensamiento, como actitudes intelectuales y como un concepto esencial que incluye la sensibilidad, inclinación y habilidad hacia una conducta y su realización (Nieto y Saiz, 2008). En este sentido, la incidencia del componente disposicional del individuo hacia el pensamiento crítico podría dar cuenta de los resultados obtenidos en el presente ejercicio investigativo y de las diferencias observadas en las puntuaciones de la muestra.

En cuanto a la relación entre el pensamiento crítico y las variables sociodemográficas, se vislumbra que los resultados antes y después del programa de intervención no difieren en función del sexo, edad, pertenencia a pueblos originarios o indígenas, procedencia de institución educacional y puntaje (PSU), aspecto que corrobora que las estrategias implementadas de meeting en cadena, triangulación transformadora y controversia permitieron el desarrollo de este tipo de pensamiento.

Específicamente, en la variable sexo se encuentran estudios que arrojaron resultados similares a los de esta investigación, al precisar que tanto hombres como mujeres desarrollan el pensamiento crítico por igual, exhibiendo las mismas capacidades y habilidades (Anganoy et al., 2017; Betancourth et al., 2020; Ennis et al., 1985; Loaiza y Osorio, 2018). En contraposición, los estudios de Beltrán y Torres (2009), Molina et al. (2016) y García y Vázquez (2017), evidenciaron que los estudiantes de sexo femenino presentan valores más altos de desarrollo de pensamiento crítico, en comparación a los resultados obtenidos por los participantes de género masculino.

Frente a la edad, autores como Aznar y Laiton (2017), Betancourth et al. (2017; 2020) develan la no existencia de diferencias entre el grupo etario y el desarrollo del atributo. Al contrario, Bejarano et al. (2014) encontraron que los estudiantes con edades inferiores a 19 años presentaron un nivel de desempeño superior en una de las habilidades de pensamiento crítico evaluadas, en contraste con los participantes de 19 años o más. Por su parte, García y Vázquez (2017) concluyeron que a mayor edad de los estudiantes, mayor puntaje en pensamiento crítico.

En relación con los antecedentes educacionales de los estudiantes, como procedencia institucional y

Pensamiento Americano Vol. 14 - No. 27 - p.p. 125-136 • 2021 • Enero-Junio Corporación Universitaria Americana • Barranquilla, Colombia • ISSN-e: 2745-1402 http://publicaciones.americana.edu.co/index.php/pensamientoamericano/index 
puntajes en pruebas de estado, los hallazgos de investigaciones en esta línea difieren de los alcanzados en el presente estudio, puesto que señalan que los estudiantes de instituciones educativas privadas reportaron puntajes más altos en pensamiento crítico que aquellos provenientes de centros educativos de carácter público (García y Vázquez, 2017; Loaiza y Osorio, 2018).

Desde esta perspectiva, la discusión precedente da pie a considerar que el fin último de los procesos educativos consiste en estimular destrezas de orden superior, indistintamente de las características sociodemográficas de los sujetos, de ahí que deba ser promovido como una competencia transversal en las diversas áreas disciplinares y profesionales en toda la población estudiantil (Molina et al., 2016).

Por último, es posible acotar que para lograr avanzar en la formación de pensar críticamente, se hace necesario articular integralmente las prácticas pedagógicas desde las cuales se estructura y desarrolla la enseñanza-aprendizaje, concibiendo que no son procesos lineales o memorísticos, sino que por el contrario demandan que el individuo confronte de forma continua su pensamiento, en aras de corregir o modificar sus razonamientos y actuar de formar consistente con estos (Carrillo, 2000; González, 2006).

\section{Referencias}

Acosta, C. (2002). Efectos del diálogo socrático sobre el pensamiento crítico en estudiantes universitarios. Psicología Desde El Caribe, 10, 1-26.

Álvarez, G. (2013). La evaluación de las habilidades del pensamiento crítico asociadas a la escritura digital. Revista Virtual Universidad Católica Del Norte, 40, 68-83.

Anganoy, A., Pantoja, C., Jurado, M., Vallejo, R. \& Botina, Z. (2017). Caracterización de las habilidades del pensamiento crítico y su relación con el desempeño académico. [Tesis de maestría, Universidad Pontificia Bolivariana]. Istname. https://repository. upb.edu.co/handle/20.500.11912/3374

Aznar, I. \& Laiton, I. (2017). Desarrollo de Habilidades Básicas de Pensamiento Crítico en el Contexto de la Enseñanza de la Física Universitaria. Formación Universitaria, 10(1), 71-78. https://doi.org/10.4067/S0718-50062017000100008

Bejarano, L. M., Galván, F. E. \& López, B. (2014). Pensamiento crítico y motivación hacia el pensamiento crítico en estudiantes de psicología. Aletheia. Revista de Desarrollo Humano, Educativo y Social Contemporáneo, 6(2), 202-223. https://doi. org/10.11600/21450366.6.2aletheia.202.223

Beltrán, M. \& Torres, N. (2009). Caracterización de habilidades de pensamiento crítico en estudiantes de educación media a través del test HCTAES. Zona Próxima, 11, 66-85.

Bernal, M., Gómez, M. \& Iodice, R. (2019). Interacción conceptual entre el pensamiento crítico y metacognición. Revista Latinoamericana de Estudios Educativo, 15(1), 193-217. https:// doi.org/10.1715l/rlee.2019.15.1.11

Betancourt, S. (2010). Congreso Iberoamericano de Educación: Metas Educativas 2021. Desarrollo Del Pensamiento Crítico a Través de La Controversia, 1-8.

Betancourth, S., Enríquez, A. \& Castillo, P. (2013). La controversia-socrática en el desarrollo del pensamiento crítico en estudiantes universitarios. Revista Virtual Universidad Católica Del Norte, 39, 71-84.

Betancourth, S., Insuasti, K. \& Riascos, N. (2012). Pensamiento crítico a través de la discusión socrática en estudiantes universitarios. Revista Virtual Católica Del Norte, 35, 147-167. 
Betancourth, S., Muñoz, K. T. \& Rosas, T. J. (2017). Evaluación del pensamiento crítico en estudiantes de educación superior de la región de Atacama-Chile. Prospectiva, 23, 199-223. https://doi.org/10.25100/prts.v0i23.4594

Betancourth, S., Paz, O. \& Obando, M. (2019). Evaluación de un programa de proyecto de vida a través de la controversia. $R e-$ vista Virtual Universidad Católica Del Norte, 57, 60-72. https://doi.org/10.35575/rvucn.n57a5

Betancourth, S., Tabares, Y., \& Martínez, V. (2020). Programa de intervención en debate crítico sobre el pensamiento crítico en universitarios. Educación y Humanismo, 22(38), 1-17. https://doi.org/10.17081/EDUHUM.22.38.3577

Betancourth, S., Zambrano, C., Ceballos, A., Moreno, J. \& Aroca, L. (2018). Adaptación y validación de un instrumento de pensamiento crítico. Universidad de Nariño.

Bezanilla, J., Poblete, M., Fernández, D., Arranz, S. y Campo, L. (2018). El Pensamiento Crítico desde la Perspectiva de los Docentes Universitarios. Estudios Pedagógicos XLIV, 1, 89-113.

Carrillo, S. (2000). Formación de herramientas científicas en el niño pequeño. Revista Latinoamericana de Psicología, 32(3), $546-548$.

Ennis, R. (1996). Critical Thinking Dispositions : Their Nature and Assessability. Informal Logic, 18(2), 165-182.

Ennis, R., Millman, J. \& Norbert, T. (1985). Cornell critical thinking tests level X \& level Z: manual. Pacific Grove, CA: Midwest Publications. https://www.worldcat.org/title/ cornell-critical-thinking-tests-level-x-level-z-manual/oclc/33987819

Espinosa, Y. C. \& Estévez, A. L. G. (2013). Auto-conocimiento de los estilos de aprendizaje, aspecto esencial en la actividad de estudio. REICE. Revista Iberoamericana Sobre Calidad, Eficacia y Cambio En Educación, 11(3), 123-137.

Facione, P. A. (2007). Pensamiento Crítico: ¿Qué es y por qué es importante? Eduteka. http://www.eduteka.org/PensamientoCriticoFacione.php

Fuentes, C. (2011). Elementos para o Desenho de um Modelo de Debate Crítico na Escola. Centro de Estudios de La Argumentación y El Razonamiento, 1-14. https://www.researchgate. net/publication/278026052
García, B. \& Vázquez, A. (2017). Relación entre el pensamiento crítico y el desempeño académico en alumnos de escuela preparatoria. Educar, 54(2), 41l. https://doi.org/10.5565/rev/ educar.768

González, J. (2006). Discernimiento: evolución del pensamiento crítico en la educación superior: el proyecto de la Universidad Icesi. Universidad Icesi.

Guevara, G. (2010). Aprendizaje basado en problemas como técnica didáctica para la enseñanza del tema de la recursividad. InterSedes: Revista de Las Sedes Regionales, 11(20), 142-167.

Halpern, D. F. (1998). Teaching critical thinking for transfer across domains: Disposition, skills, structure training, and metacognitive monitoring. American Psychologist, 53(4), 449-455. https://doi.org/10.1037/0003-066X.53.4.449

Henríquez, G., Higuera, V., Rosano, E. y Robles, N. (2019). Estrategias de formación en busca de una Universidad socialmente responsable. Pensamiento Americano, 12(24), 180-197. https://doi.org/10.21803/pensam.v12i24.335

Hernández, R., Fernández, C. \& Baptista, M. (2014). Metodología de la investigación. Mc Graw Hill (6ta edició).

Hincapie, D., Ramos, A. \& Chirino, V. (2017). Aprendizaje Basado en Problemas como estrategia de Aprendizaje Activo y su incidencia en el rendimiento académico y Pensamiento Crítico de estudiantes de Medicina. Revista Complutense de Educación, 29(3), 665-681.https://doi.org/10.5209/RCED.53581

Jaimes, A. (2016). Impacto de un programa de pensamiento crítico en estudiantes de un liceo de la Región del Biobío. Pensamiento Educativo: Revista de Investigación Educacional Latinoamericana, 53(2), 1-11. https://doi.org/10.7764/ PEL.53.2.2016.6

Lazo, L. \& Herrera, H. (2011). Aplicación de un modelo de intervención pedagógica que desarrolla estrategias de pensamiento crítico para estudiantes de carreras del área de las ciencias. Diálogos Educativos, 21, 81-97.

Leitão, S., De Chiaro, S. \& Cano, M. (2016). El debate crítico: un recurso de construcción del conocimiento en el aula. Textos. Didáctica de La Lengua y La Literatura, 73, 26-33.

Loaiza, Y. \& Osorio, L. (2018). El desarrollo de pensamiento crítico en ciencias naturales con estudiantes de básica secundaria 
en una Institución Educativa de Pereira - Risaralda. Diálogos Sobre Educación. Temas Actuales En Investigación Educativa, 9(16), 1-24.

Macedo, A. (2018). Pensamiento crítico y rendimiento académico en los ingresantes del curso de Estadística I en la Facultad de Ingeniería Económica, Estadística y Ciencias Sociales. Universidad Nacional de Ingeniería - 2017. [Tesis de maestría, Universidad Peruana Cayetano Heredia]. https://repositorio. upch.edu.pe/handle/20.500.12866/1541

Molina, C., Morales, G. \& Valenzuela, J. (2016). Competencia transversal pensamiento crítico: Su caracterización en estudiantes de una secundaria de México. Revista Electrónica Educare, 20(1), 1-26. https://doi.org/10.15359/ree.20-1.11

Montoya, J. \& Monsalve, J. C. (2008). Estrategias didácticas para fomentar el pensamiento crítico en el aula. Revista Virtual Universidad Católica Del Norte, 25, 1-25.

Núñez, S., Ávila, J. \& Olivares, S. (2017). El desarrollo del pensamiento crítico en estudiantes universitarios por medio del aprendizaje basado en problemas. Revista Iberoamericana de Educación Superior, 8(23), 84. https://doi.org/10.22201/ iisue.20072872e.2017.23.3012

Ossa, C., Palma, M., Martín, N. \& Díaz, C. (2018). Evaluación del pensamiento crítico y científico en estudiantes de pedagogía de una universidad chilena. Revista Electrónica Educare, 22(2), 1-18. https://doi.org/10.15359/REE.22-2.12

Palacios, W., Alvarez, M., Moreira, J. \& Moran, C. (2017). Una mirada al pensamiento crítico en el proceso docente educativo de la educación superior. EDUMECENTRO, 9(4), 194-206.

Paul, R. y Elder, L. (2002). Critical Thinking: Tools for Taking Charge of Your Professional and Personal Life. En Financial Times Prentice Hall. http://www.bisenter.com

Paul, R. y Elder, L. (2003). La mini-guía para el Pensamiento crítico Conceptos y herramientas. En Fundación para el Pensamiento Crítico. https://www.criticalthinking.org/resources/ PDF/SP-ConceptsandTools.pdf

Pineda, M., \& Cerrón, A. (2015). Pensamiento crítico y rendimiento académico de estudiantes de la Facultad de Educación de la Universidad Nacional del Centro del Perú. Horizonte de La Ciencia, 5(8), 105-110.
Robles, S., Cisneros, L. y Guzmán, C. (2016). Evaluación del nivel de pensamiento crítico en estudiantes universitarios de pregrado y posgrado. El caso de un Centro Universitario Temático de la Universidad de Guadalajara. Revista de Educación y Desarrollo, 39, 63-71.

Saiz, C. \& Rivas, S. F. (2011). Evaluation of the ARDESOS program: An initiative to improve critical thinking skills. Journal of the Scholarship of Teaching and Learning, 11(2), 34-51.

Sánchez, C. S. \& Rivas, S. F. (2012). Pensamiento crítico y aprendizaje basado en problemas cotidianos. REDU. Revista de Docencia Universitaria, 10(3), 325-346. https://doi. org/10.4995/REDU.2012.6026

Sastoque, D., Ávila, J. \& Olivares, S. (2016). Aprendizaje Basado en Problemas para la construcción de la competencia del Pensamiento Crítico. Voces y Silencios. Revista Latinoamericana de Educación, 7(1), 148-172. https://doi.org/10.18175/ vys7.1.2016.08

Steffens, E., Ojeda, D., Martínez, J., Hernández, H. \& Moronta, Y. (2018). Presencia del pensamiento crítico en estudiantes de educación superior de la Costa Caribe Colombiana. Revista Espacios, 39(30), 1-14.

Tünnermann, C. (2003). La Universidad Latinoamericana ante los retos del siglo XXI. En Colección UDUAL. https://www.yumpu.com/es/document/view/14139844/la-universidad-latinoamericana-ante-los-retos-udual

Vilà, M., Palou, J., Grau, M., Castella, J., Ballesteros, C. \& Cros, A. (2005). El discurso oral formal: Contenidos de aprendizaje y secuencias didácticas. En Contenidos de aprendizaje y secuencias didácticas. https://books.google.com/books/about/ EL_discurso_oral formal.html?hl=es\&id=XvZpBisBCFUC

Villarroel, V. \& Bruna, D. (2014). Reflexiones en torno a las competencias genéricas en educación superior: Un desafío pendiente. Psicoperspectivas. Individuo y Sociedad, 13(1), 23-34. https://doi.org/10.5027/psicoperspectivas-Voll3-Issuel-fulltext-335

2021, Vol. 14(27) 125-136. (C)The Author(s) 2021 Reprints and permission: www.americana.edu.co http://publicaciones.americana.edu.co/index.php/pensamientoamericano/index 